\title{
A Hemorrhagic Pineal Cyst with a Bacterial Meningitis-like Manifestation and Benign Outcome
}

\author{
Kanji Yamamoto, Toshikazu Omodaka, Rie Watanabe and Minori Kodaira
}

\begin{abstract}
Pineal cysts are a common incidental finding in imaging studies, and the majority of such cysts are asymptomatic. However, hemorrhaging pineal cysts, which are considered to be rare, are often associated with severe symptoms. We herein describe the case of a 58-year-old patient with the novel manifestation of a bleeding pineal cyst, who had a benign outcome without any surgical treatment. Although the clinical manifestations resembled those of bacterial meningitis, magnetic resonance images suggested chemical meningitis caused by an intracystic hemorrhage and rupture of the pineal cyst.
\end{abstract}

Key words: pineal cyst, pineal apoplexy, chemical meningitis

(Intern Med 52: 2817-2820, 2013)

(DOI: 10.2169/internalmedicine.52.0451)

\section{Introduction}

Pineal cysts are a common incidental finding in imaging studies, especially since the advent of magnetic resonance (MR) imaging (1). Although the majority of these cysts are benign and are usually asymptomatic, some are occasionally associated with headaches, hydrocephalus, extraocular movement abnormalities and Parinaud's syndrome $(2,3)$. The hemorrhage of a pineal cyst, which is sometimes referred to as pineal apoplexy, is considered to be rare and is often associated with severe symptoms (4-6). We herein describe an adult case of chemical meningitis induced by an intracystic hemorrhage of the pineal region in which the patient presented with clinical manifestations resembling bacterial meningitis and was able to achieve a benign outcome without any surgical treatment.

\section{Case Report}

A 58-year-old previously healthy man had a headache for two days that recovered spontaneously. One week later, he presented with a throbbing headache and was examined in a hospital. Rizatriptan was prescribed; however, it was not effective. The next day, the patient developed a fever. The headache worsened, and he was admitted to our hospital.
His body temperature was $37.3^{\circ} \mathrm{C}$. A physical examination showed no signs of focal infection or inflammation. He was alert, and no meningeal irritation, papilledema, ophthalmoplegia, gaze disturbances or other abnormalities were found on neurological examinations. Routine blood examinations showed no diabetes, with a leukocyte count of $10,040 / \mathrm{mm}^{3}$ containing $70.5 \%$ neutrophils and a C-reactive protein level of $8.41 \mathrm{mg} / \mathrm{dL}$. Serological tests for syphilis, human immunodeficiency virus and antinuclear antibodies were negative. Examinations of the cerebrospinal fluid (CSF) revealed xanthochromia and a nucleated cell count of $1,072 / \mathrm{mm}^{3}$ that consisted of $71 \%$ polymorphonuclear leukocytes. The concentrations of total protein and lactate dehydrogenase in the $\mathrm{CSF}$ were increased $(93 \mathrm{mg} / \mathrm{dL}$ and $144 \mathrm{IU} / \mathrm{L}$, respectively), and the CSF glucose level was slightly decreased (48 $\mathrm{mg}$ / $\mathrm{dL}$; the blood glucose level was $105 \mathrm{mg} / \mathrm{dL}$ at that point). Routine blood and CSF bacterial cultures produced no growth. Cranial computed tomography (CT) revealed an oval cyst with calcification partially along the rim in the pineal region without ventriculomegaly (Fig. A). MR imaging demonstrated that the cyst measured $19 \times 14 \times 10 \mathrm{~mm}$. The major portion of the contents was homogenous and hyperintense on T2-weighted images (Fig. B), slightly hyperintense compared to the CSF on T1-weighted images (Fig. C) and fringed by a partially gadolinium-enhanced thin rim (Fig. D). Although the cyst also exhibited a fluid-fluid level 

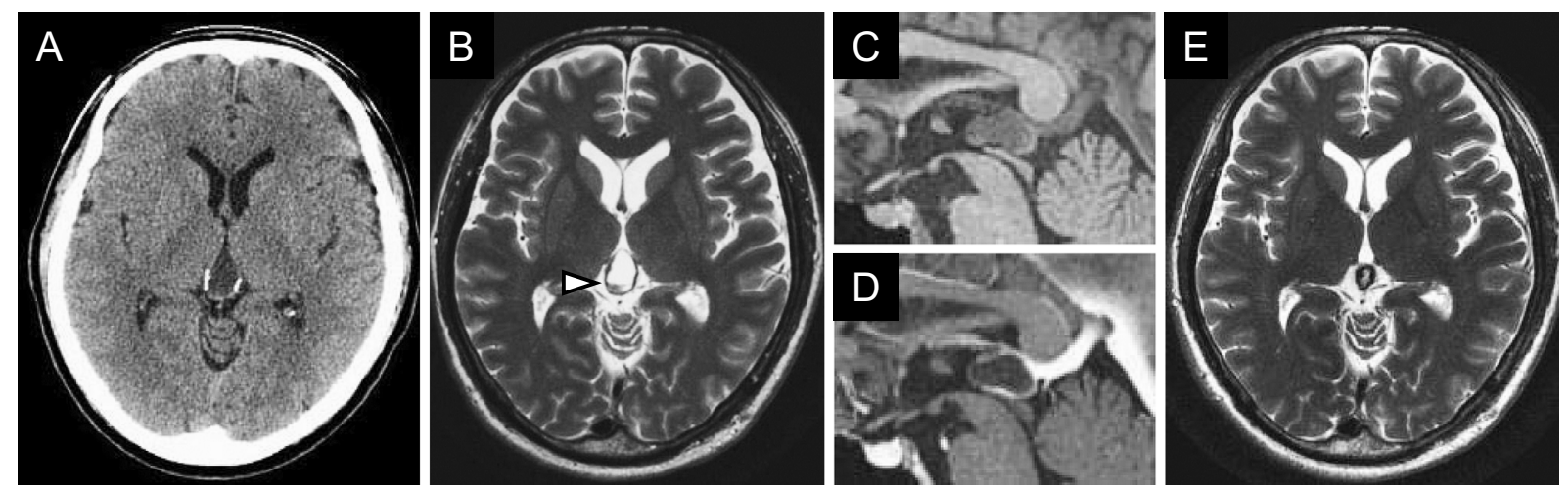

Figure. Images obtained on admission (A-D). Noncontrast computed tomographic images revealed an oval cyst in the pineal region without ventriculomegaly. The pineal cyst exhibited calcification partially along the rim (A). On magnetic resonance imaging, the major portion of the contents of the cyst was homogenous and hyperintense on T2-weighted images (B), slightly hyperintense compared to the cerebrospinal fluid on $\mathrm{T} 1$-weighted images $(\mathrm{C})$, and fringed by a partially gadolinium-enhanced thin rim (D). The cyst also exhibited a fluid-fluid level, suggesting intracystic hemorrhage (B, arrowhead). A magnetic resonance image (T2-weighted image) obtained two and a half months after the first image showed the collapsed pineal cyst and an extremely low signal along the rim of the residual cyst, thereby indicating the presence of an intracystic hemorrhage and subsequent hemosiderin deposition (E).

(Fig. B), thus suggesting an intracystic hemorrhage, we overlooked this finding at that time. The imaging studies showed no obvious findings of intracranial infection. Initially, we suspected bacterial meningitis and intravenously administered $8 \mathrm{~g}$ /day of ampicillin, $8 \mathrm{~g} /$ day of cefotaxime and $2 \mathrm{~g}$ /day of vancomycin, adding $6.6 \mathrm{mg} /$ day of dexamethasone for the first three days. The patient became afebrile, and his headache rapidly disappeared. Follow-up CSF examinations showed a lowered nucleated cell count and protein level. Following the administration of antimicrobials for three weeks, he left the hospital without any symptoms. Two months later, a cranial MR image revealed that the pineal cyst had collapsed, thus suggesting leakage of the intracystic fluid. A T2-weighted image demonstrated an extremely low signal along the rim of the residual cyst, indicating an intracystic hemorrhage and subsequent hemosiderin deposition (Fig. E).

\section{Discussion}

Pineal cysts are a common incidental finding on MR images. The prevalence of pineal cysts in patients undergoing intracranial imaging has been estimated to range from 1.0 to $4.4 \%(1,7,8)$. The prevalence of pineal cysts has a female predominance and an age-related pattern, with the prevalence rising to a peak late in childhood or early in adulthood and then falling throughout the later age ranges $(1,8)$. Furthermore, autopsy series have suggested a far higher prevalence $(21-41 \%)$ of pineal cysts $(9,10)$. Although the vast majority of pineal cysts, even large ones, can be expected to be found incidentally and remain asymptomatic (1), some patients may present with signs and symptoms of increased intracranial pressure or focal signs and symptoms due to compression and edema in neighboring structures $(2,3)$. Pineal apoplexy is generally considered to be rare $(1,4-6)$. Most patients with pineal apoplexy have been reported to have relatively severe symptoms that often accompany acute occlusive hydrocephalus (4-6) and may occasionally result in sudden death (11) or the need for surgical treatment. In contrast, there have been few reports of patients with asymptomatic intracystic hemorrhage (7) or spontaneous resolution of hemorrhagic pineal cysts $(4,12,13)$.

In our patient, the meningitis and intracystic bleeding of the pineal cyst occurred almost simultaneously, and then the cyst collapsed within a short period of time. These consecutive events are not likely to occur accidentally. Antecedent bacterial meningitis may have induced the intracystic hemorrhage, rupture and collapse of the pineal cyst. For the following reasons, however, we do not support this possibility. First, there was no evidence of bacterial infection. Clinical examinations showed no findings of infection of the structures that neighbored the meninges, such as sinusitis or otitis media, or an immunocompromised state. Although the patient had not received antibiotics at any time prior to admission, his blood and CSF bacterial cultures produced no growth. Furthermore, his recovery may have been too brisk for bacterial meningitis. Second, it is hard to reasonably conclude that meningeal inflammation caused the bleeding inside of the capsulated cyst. Because the mildly gadolinium-enhanced peripheral rim on the initial MR images did not show the involvement of inflammation, but rather the common appearance of pineal cysts $(1,3)$, the result did not suggest that the inflammation was especially strong around the cyst. Third, the intracystic hemorrhage seems to have preceded the meningitis. On admission, MR imaging showed a fluid-fluid level, suggesting a recent intra- 
cystic hemorrhage $(4,6,7)$; however, the CT images did not demonstrate obvious high density within the cyst. The images implied the presence of bleeding in the subacute stage or recurrent minimal hemorrhage. The initial headache, which occurred 10 days before admission, may possibly have reflected the intracystic hemorrhage.

The rupture of various cranial and/or spinal tumors harboring a liquid component and the release of their contents into the CSF may cause sterile chemical meningitis that mimics viral or bacterial meningitis or Mollaret's meningitis. These tumors include craniopharyngioma cysts, dermoid or epidermoid cysts (14), and Rathke cleft cysts (15). Furthermore, there are several reports of patients with hemorrhagic pituitary adenoma (pituitary adenoma apoplexy) with symptoms mimicking bacterial meningitis (16-20). In these cases, the clinical manifestations of meningitis were explained by the release of necrotic and hemorrhagic material into the subarachnoid space. It is interesting to note that, on imaging, the contents of the cystic lesions were found to be intact in a considerable number of patients with cystic tumors who already had chemical meningitis (17, 19-21). These facts possibly indicate that either a very slow or a small amount of leakage from a cyst can cause chemical meningitis. Kitayama et al. reported the case of a 40-yearold woman with recurrent aseptic meningitis and mononuclear CSF pleocytosis that was suspected to have been caused by the rupture of a pineal cyst, although imaging studies showed no evidence of intracystic hemorrhage (22). To the best of our knowledge, this is the only report in which meningitis developed in association with a pineal cyst.

We surmised that, in our patient, the hemorrhage in the pineal cyst could have caused the leakage of the intracystic content into the CSF, thus inducing the chemical meningitis with xanthochromia. Old and recent areas of hemorrhage were identified microscopically in the resected pineal cyst of a patient with pineal apoplexy (23). The release of the debris accumulated by a single or recurrent intracystic hemorrhage seems to be able to cause meningitis with polymorphonuclear CSF pleocytosis. In the present case, the second MR images obtained 2.5 months after the first images showed involution and hemosiderin deposition in the pineal cyst. After assessing the above-mentioned cases of cystic tumors with chemical meningitis, it is very likely that shrinking of the hemorrhagic cyst took many days. Therefore, it can be speculated that the contents of the cyst had already leaked out before the first images were acquired, even if the cyst remained apparently intact. By obviating acute occlusive hydrocephalus, the rupture of the cyst presumably led to a favorable outcome. In addition, the administered dexamethasone may have been effective against the meningitis due to its anti-inflammatory and immunosuppressive actions (14).

Asymptomatic enlargement has been identified in some pineal cysts (1). Several hypotheses, including growth due to intracystic microhemorrhage $(2,3,5)$, have been proposed to explain the enlargement of pineal cysts. There is the possibility that asymptomatic or mildly symptomatic intracystic hemorrhages may not be as rare as previously thought, and, if so, the term pineal apoplexy may no longer be suitable for describing bleeding in a pineal cyst. Therefore, given the fact that the prevalence of pineal cysts is extremely high, it is necessary to consider a diagnosis of chemical meningitis caused by intracystic hemorrhage of the pineal gland in patients with meningitis of unknown etiology or meningitis that is thought to be caused by microbial infection when pathogenic microorganisms cannot be identified.

\section{The authors state that they have no Conflict of Interest (COI).}

\section{References}

1. Al-Holou WN, Terman SW, Kilburg C, et al. Prevalence and natural history of pineal cysts in adults. J Neurosurg 115: 1106-1114, 2011.

2. Klein P, Rubinstein LJ. Benign symptomatic glial cysts of the pineal gland: a report of seven cases and review of the literature. J Neurol Neurosurg Psychiatry 52: 991-995, 1989.

3. Fain JS, Tomlinson FH, Scheithauer BW, et al. Symptomatic glial cysts of the pineal gland. J Neurosurg 80: 454-460, 1994.

4. Koenigsberg RA, Faro S, Marino R, Turz A, Goldman W. Imaging of pineal apoplexy. Clin Imaging 20: 91-94, 1996.

5. McNeely PD, Howes WJ, Mehta V. Pineal apoplexy: is it a facilitator for the development of pineal cysts? Can J Neurol Sci 30: 67-71, 2003.

6. Sarikaya-Seiwert S, Turowski B, Hänggi D, Janssen G, Steiger HJ, Stummer W. Symptomatic intracystic hemorrhage in pineal cysts. Report of 3 cases. J Neurosurg Pediatr 4: 130-136, 2009.

7. Mamourian AC, Towfighi J. Pineal cysts: MR imaging. AJNR Am J Neuroradiol 7: 1081-1086, 1986.

8. Sawamura Y, Ikeda J, Ozawa M, Minoshima Y, Saito H, Abe H. Magnetic resonance images reveal a high incidence of asymptomatic pineal cysts in young women. Neurosurgery 37: 11-16, 1995.

9. Tapp E, Huxley M. The histological appearance of the human pineal gland from puberty to old age. J Pathol 108: 137-144, 1972.

10. Hasegawa A, Ohtsubo K, Mori W. Pineal gland in old age; quantitative and qualitative morphological study of 168 human autopsy cases. Brain Res 409: 343-349, 1987.

11. Richardson JK, Hirsch CS. Sudden, unexpected death due to "pineal apoplexy". Am J Forensic Med Pathol 7: 64-68, 1986.

12. Nimmagadda A, Sandberg DI, Ragheb J. Spontaneous involution of a large pineal region hemorrhagic cyst in an infant. Case report. J Neurosurg 104 (4 Suppl): 275-278, 2006.

13. Ayhan S, Bal E, Palaoglu S, Cila A. Pineal cyst apoplexy: report of an unusual case managed conservatively. Neurol Neurochir Pol 45: 604-607, 2011.

14. Lunardi $P$, Missori $P$. Cranial and spinal tumors with meningitic onset. Ital J Neurol Sci 11: 145-151, 1990.

15. Chaiban JT, Abdelmannan D, Cohen M, Selman WR, Arafah BM. Rathke cleft cyst apoplexy: a newly characterized distinct clinical entity. J Neurosurg 114: 318-324, 2011.

16. Bjerre P, Lindholm J. Pituitary apoplexy with sterile meningitis. Acta Neurol Scand 74: 304-307, 1986.

17. Jassal DS, McGinn G, Embil JM. Pituitary apoplexy masquerading as meningoencephalitis. Headache 44: 75-78, 2004.

18. Brouns R, Crols R, Engelborghs S, De Deyn PP. Pituitary apoplexy presenting as chemical meningitis. Lancet 364: 502, 2004.

19. Huang WY, Chien YY, Wu CL, Weng WC, Peng TI, Chen HC. Pi- 
tuitary adenoma apoplexy with initial presentation mimicking bacterial meningoencephalitis: a case report. Am J Emerg Med 27: 517, 2009.

20. Paisley AN, Syed AA. Pituitary apoplexy masquerading as bacterial meningitis. CMAJ 184: 1812, 2012.

21. Rajput D, Srivastva A, Kumar R, Mahapatra A. Recurrent chemical meningitis in craniopharyngioma without reduction in size of cyst: case report of two cases and review of the literature. Turk
Neurosurg 22: 233-236, 2012.

22. Kitayama J, Toyoda K, Fujii K, et al. Recurrent aseptic meningitis caused by rupture of a pineal cyst. No To Shinkei (Bain Nerve) 48: 1147-1150, 1996 (in Japanese, Abstract in English).

23. Apuzzo ML, Davey LM, Manuelidis EE. Pineal apoplexy associated with anticoagulant therapy. Case report. J Neurosurg 45: 223226, 1976.

(C) 2013 The Japanese Society of Internal Medicine http://www.naika.or.jp/imonline/index.html 